\title{
Advances in minimally invasive surgery for hepatocellular carcinoma
}

\author{
Cun-Zhen Zhang, Nan Li \\ Department of Hepatic Surgery, Shanghai Eastern Hepatobiliary Surgery Hospital, Shanghai 200438, China.
}

Correspondence to: Dr. Nan Li, Department of Hepatic Surgery, Shanghai Eastern Hepatobiliary Surgery Hospital, 255 Changhai Road, Yangpu District, Shanghai 200438, China. E-mail: liparislisi@aliyun.com

How to cite this article: Zhang CZ, Li N. Advances in minimally invasive surgery for hepatocellular carcinoma. Hepatoma Res 2020;6:77. http://dx.doi.org/10.20517/2394-5079.2020.74

Received: 30 Jul 2020 First Decision: 17 Sep 2020 Revised: 14 Oct 2020 Accepted: 26 Oct 2020 Published: 6 Nov 2020

Academic Editor: Ho-Seong Han Copy Editor: Cai-Hong Wang Production Editor: Jing Yu

\begin{abstract}
Hepatocellular carcinoma (HCC) is the fifth most common cancer and the third leading cause of cancer-related death worldwide. Surgical resection is still regarded as the first choice of treatment for HCC. With advances in technology and techniques, minimally invasive surgery has now become the standard of care in almost every field in general surgery, including hepatectomy surgery. This review focuses on the latest advances in minimally invasive surgical treatment of HCC, including laparoscopic hepatectomy $(\mathrm{LH})$, robotic hepatectomy $(\mathrm{RH})$ and other minimally invasive treatment technologies. Although some limitation in $\mathrm{LH}$ or $\mathrm{RH}$ exists, these minimally invasive techniques may be performed for hepatectomy with benefits, and have a promising future. With the development of technology and the improvement of surgical operations, patients will benefit from this.
\end{abstract}

Keywords: Hepatocellular carcinoma, minimally invasive surgery, laparoscopic hepatectomy, robotic hepatectomy

\section{INTRODUCTION}

Hepatocellular carcinoma (HCC) is the fifth most common cancer and the third leading cause of cancerrelated death worldwide ${ }^{[1-4]}$. Because its early symptoms are not typical, most liver cancers clinically diagnosed belong to the advanced stage ${ }^{[5]}$. In addition, most of these patients are accompanied by portal vein tumor thrombus or pulmonary metastasis ${ }^{[6,7]}$. Surgical resection is still regarded as the first choice of HCC treatment ${ }^{[8-12]}$. However, due to intrahepatic metastasis and early recurrence, postoperative patients' prognosis is poor ${ }^{[13-18]}$, and the 5-year survival rate is relatively low ${ }^{[19]}$. The high recurrence and vascular 
invasion of HCC limit the long-term survival of HCC patients. The recurrence rate of HCC is as high as $80 \%$ within 5 years after radical resection ${ }^{[20]}$. However, due to a relatively insidious onset, most of the patients are in the middle and late stages when they seek medical treatment, with only $10 \%$ at a stage amenable to surgical intervention ${ }^{[21]}$. Moreover, the postoperative recurrence rate is high. In recent years, a variety of new technologies and therapies have emerged constantly, which have posed a challenge to the surgical resection of HCC. In addition to liver transplantation, surgical resection of tumor has been accepted as an effective local treatment for HCC. The innovation of HCC treatment technology and the progress of surgical technology have improved the safety of HCC resection ${ }^{[22]}$.

With advances in technology and techniques, minimally invasive surgery has now become the standard of care in almost every field in general surgery, including hepatectomy surgery. Since the first laparoscopic pericystectomy for hepatic echinococcosis carried out in France in 1991, there have been increasing reports on the laparoscopic treatments for HCC. The da Vinci Surgical System (Intuitive Surgical Inc., United States) is an advanced and minimally invasive surgery tool, which has been shown to have advantages in complex hepatectomy. Liu et al. ${ }^{[23]}$ formulated international consensus statement on robotic hepatectomy surgery. On the basis of ensuring the therapeutic effect and safety, the development of new surgical treatment is conducive to the prognosis of patients and the development of the whole treatment system.

This review focuses on the latest advances in minimally invasive surgical treatment of HCC, including laparoscopic hepatectomy ( $\mathrm{LH})$, robotic liver resection and other minimally invasive treatment technologies.

\section{LAPAROSCOPIC HEPATECTOMY}

As far as the traditional treatment viewpoint is concerned, surgical resection is the first choice for the treatment of liver cancer ${ }^{[24]}$. With the update of treatment viewpoints and the innovation of technical equipment, laparoscopic technology is widely used in various surgical treatment fields. LH as one of the minimally invasive treatment methods for hepatocellular carcinoma, is increasingly used in clinical surgery by more and more surgeons. In 1991, Reich et al. ${ }^{[25]}$ completed the first laparoscopic hepatectomy in the world, and the laparoscopic technique was gradually applied to the surgical treatment of hepatobiliary diseases. In 1994, Zhou et al. ${ }^{[26]}$ reported the first laparoscopic hepatectomy in China. Owing to the differences and gaps in the mastery of technical difficulties and risk awareness, the rich liver blood flow, laparoscopic hepatic portal occlusion that is more difficult than laparotomy, and lack of open operation feel and rapid reactions, accurate hemostasis and control of bleeding are the key to the success of laparoscopic hepatectomy, so the clinical development of laparoscopic hepatectomy is still in the development stage. The classification of laparoscopic hepatectomy in domestic and foreign guidelines mainly includes pure laparoscopic liver resection, hand-assisted laparoscopic liver resection and laparoscopic assisted hepatectomy (hybrid procedures). The indications of laparoscopic hepatectomy vary globally. In 2013, the domestic expert consensus ${ }^{[2]}$ proposed that the indications of laparoscopic hepatectomy include symptomatic benign diseases, intra-and extra-hepatic bile duct calculi and liver malignant tumors. However, there are still controversies about laparoscopic hepatectomy. According to a survey conducted in Japan in 2013, more than half of the surveyed medical centers routinely perform laparoscopic hepatectomy, but the indications are not consistent, and preoperative liver function reserve and intraoperative bleeding control occupy the primary position. More than two-thirds of medical centers hold that laparoscopic hepatectomy can be used for living donor liver transplantation ${ }^{[28]}$. Limited by the surgical instrument and technical mastery, small-scale hepatectomy or anatomical hepatectomy can be performed if the lesion location of liver cancer is superficial and limited, and does not invade large blood vessels and bile ducts according to the traditional surgical viewpoint ${ }^{[28]}$. Due to the limitation of the laparoscopic visual field and operation equipment activities, hand-assisted laparoscopic liver resection or laparotomy is the best choice for some larger tumors requiring complex hepatectomy or large-scale hepatectomy. At present, the 
industry still maintains a cautious attitude towards the transition to laparotomy because intraoperative bleeding is difficult to effectively control for the safe integration of laparoscopic large-scale hepatectomy or postoperative bleeding. Recent evidence from some centers has shown that laparoscopic hepatectomy is acceptable for HCC patients who meet the surgical criteria, and there is no significant difference in postoperative survival time ${ }^{[29,30]}$. Rao et al. ${ }^{[31]}$ compared 700 patients who underwent pure laparoscopic liver resection and laparotomy. Compared with laparotomy, the overall incidence of complications after LH was lower and the length of stay was shorter. For liver cancer patients with obvious liver cirrhosis, the studies from Memeo et al. ${ }^{[32]}$ showed that, compared with laparotomy, patients who underwent laparoscopic liver resection had shorter operation times and complete surgical margins, but there was no significant difference in overall survival rate and disease-free survival time. The 1-, 5-, and 10-year survival rate of laparoscopic liver resection (LLR) group reached $88 \%, 59 \%$, and $12 \%$, and that of open liver resection (OLR) group was $63 \%, 44 \%$, and $22 \%$, respectively $(P=0.27)$. However, there is no evidence that patients with liver cancer who undergo laparoscopic liver resection are afforded a better prognosis. In a recent study, Ahmed El-Gendi et al. ${ }^{[33]}$ compared the therapeutic effects of OLR with LLR in patients with liver cirrhosis (Child A) with solitary small $(<5 \mathrm{~cm})$ peripheral HCC. The results showed that LLR had significantly less operative time $(120.32 \pm 21.58 \mathrm{~min} v s .146 .80 \pm 16.59 \mathrm{~min}, P<0.001)$ and shorter duration of hospital stay $(2.40 \pm$ 0.58 days $v s .4 .28 \pm 0.79$ days, $P<0.001)$, with comparable overall complications $(25 \% v s .28 \%, P=0.02)$. LLR had comparative resection time ( $66.56 \pm 23.80 \mathrm{~min} v s .59 .56 \pm 14.74 \mathrm{~min}, P=0.218)$, amount of blood loss $(250 \mathrm{~mL} v s .230 \mathrm{~mL}, P=0.915)$, transfusion rate $(P=1.00)$, and Ro resection rate when compared with OLR. There was no significant difference between the two groups in terms of postoperative tumor-free survival time ${ }^{[33]}$. In a Japanese study, information on patients undergoing liver cancer surgery was collected from 31 centers between 2000 and 2010, and divided into LLR $(n=436)$ and OLR $(n=2969)$ groups. 387 patients were matched by propensity score matching. There were no significant differences in overall survival and disease-free survival between LLR and OLR. Patients undergoing LLR have shorter hospital stays (13 days vs. 16 days, $P<0.001)$ and fewer postoperative complications $(6.7 \% \text { vs. } 13.0 \%, P=0.003)^{[34]}$.

\section{ROBOTIC LIVER RESECTION}

With the progress and development of technology, the surgical treatment of liver cancer has gradually entered the era of minimally invasive precision treatment, mainly including laparoscopic and robotic minimally invasive treatment. The development of minimally invasive surgical techniques for liver tumors is limited by the characteristics of its own organs, a crisp texture, abundant blood supply, high numbers of structural variations of blood vessels and bile ducts, and a close relationship with surrounding organs. Although laparoscopic liver resection has made great progress on the basis of the improvement of laparotomy, it is easy to cause clamping or traction bleeding when the deep lesions and special liver segments are exposed. Therefore, the control of intraoperative bleeding, the exposure of the operative visual field and the effective hemostasis of liver section are the key points of minimally invasive surgery, and also the biggest problem of laparoscopic liver resection ${ }^{[35,36]}$. In this case, the robot assisted system is gradually improved. In 2000, Da Vinci robot assisted surgery system was approved for clinical use. It is one of the most mature surgical auxiliary operating systems, which is widely used in the field of surgery. With its unique advantages, it gradually fills the gap in the minimally invasive field of liver tumor treatment. Traditional laparoscopic liver resection has some problems, such as inflexible fixation of the operating instruments and a large swing of the visual field leverage effect ${ }^{[37,38]}$. The robot assisted surgery system has no such limitations, and the highly bionic operation can greatly simulate the fine operation of the open hand, provide stable, fixed strength and angle traction and exposure, and even surpass the human operation to a certain extent ${ }^{[39]}$, and complete the accurate resection and suturing of malignant liver tumors ${ }^{[40]}$. In 2003, Giulianotti et al ${ }^{[38]}\{$ Giulianotti, $2003 \# 170\}$ reported the first robotic liver resection in the world. Under this background, robotic surgery technology has been developing rapidly. At present, the Da Vinci surgical assistant system is widely used in various benign and malignant liver diseases, including for the indications of laparoscopic hepatectomy ${ }^{[41]}$. For some special liver segment tumors, robot assisted surgery 
is more advantageous. Traditionally, it has been considered that if the tumor located in a location difficult to dissect, intraoperative hemorrhage is anticipated to be difficult to control, or the tumor is large, these are relative contraindications to the use of minimally invasive surgery. The advantages of robot assisted surgery system can solve these problems, and the operation position is no longer the taboo of minimally invasive surgery. For tumors in the upper right posterior lobe or caudate lobe of the liver, robot assisted surgery has a better visual field than laparoscopic liver resection, and the process is more $\operatorname{smooth}^{[22,43]}$. Hu et al. ${ }^{[37]}$ performed a meta-analysis of 17 case-control studies with different surgical methods, including 487 robotic cases and 902 laparoscopic cases. The results showed that the amount of blood loss in robotic liver resection was significantly increased and the operation time was significantly longer than that in traditional laparoscopic surgery, and there was no significant difference in blood transfusion volume and blood transfusion rate between 33 cases with robotic surgery and 25 cases with laparoscopic liver resection. There was no significant difference in hospital stay, conversion rate to laparotomy, Ro resection rate, complications, or mortality between the two groups. Zhao et al. ${ }^{[4]}$ reported that successful robotic radical resection of hepatic echinococcosis located in posterosuperior liver segments, and robotic isolated partial and complete hepatic caudate lobectomy ${ }^{[45]}$. In addition, Hu et al. ${ }^{[46]}$ indicated that robotic and laparoscopic hemi-hepatectomies were associated with less intraoperative blood loss, better postoperative recovery and a lower pain score. Compared with laparoscopic hemi-hepatectomy, robotic hemi-hepatectomy was associated with significantly less intraoperative blood loss and a shorter operative time. In terms of economic benefits, the medical cost of laparoscopic hepatectomy was relatively lower. There was no significant difference in 5-year overall survival rate and disease-free survival rate between the two groups ( $65 \% v s .48 \%, 42 \% v s .38 \%$ ). Therefore, the high cost of treatment, the high requirements of operation technology and the difficulty in popularizing technical equipment may be the biggest shortcomings in the development of robotic surgery.

\section{CONCLUSION}

We have presented a comparison of published evidence for open, laparoscopic, and robotic liver resection, including systematic review and meta-analysis evidence summarizing the advantages and disadvantages of the three techniques, One of the analyses included 26 studies, including 2,630 patients (950 robotic laparoscopic surgery and 1,680 laparoscopic patients). The amount of bleeding in patients undergoing robotic hepatectomy was significantly reduced $(286 \mathrm{~mL} v s .301 \mathrm{~mL}, P<0.001)$. There is no significant difference in postoperative complications of the various surgical methods. However, there is one thing worthy of our attention that patients undergoing robotic liver resection have a higher readmission rates $(P=0.005)$, Compared with traditional open surgery, minimally invasive surgery (robot and laparoscopic) is more expensive, but there is no statistical difference. It can significantly reduce intraoperative blood loss and shorten the length of stay; the tumor volume of patients undergoing laparoscopic liver resection was significantly reduced than patients in the open surgery group ${ }^{[47-49]}$. Minimally invasive surgery for HCC is not a simple minimally invasive technology, but a process of minimally invasive concept and comprehensive treatment. For example, the ALPPS procedure (associating liver partition and portal vein ligation for staged hepatectomy) was proposed by Schnitzbauer et al. ${ }^{[50]}$ in 2015 . In order to obtain sufficient liver function reserve, create conditions for surgery, and solve the problem of insufficient residual liver volume, it is necessary to make the impossible possible. However, because of its higher rate of complications, the mortality rate is as high as $12 \%$, and, therefore, this kind of operation has caused great controversy. Currently, there is no clear clinical data to support its effect, as well as many postoperative complications, which cannot be popularized in clinical practice. Other studies have shown that the recurrence rate of ALPPS is increased ${ }^{[51]}$. Moreover, the key objective of ALPPS is to block the blood supply of the tumor, promote the growth of residual liver, and avoid postoperative liver failure. However, PVE can play a similar role in clinical practice. At present, there is no clinical evidence to prove the overall prognosis of ALPPS. With the development of technology and the improvement of surgical techniques, patients will increasingly benefit from minimally invasive surgical precision treatments for liver pathologies. 


\section{DECLARATIONS}

\section{Authors' contributions}

Conceived the review: Zhang CZ, Li N

\section{Availability of data and materials}

Not applicable.

\section{Financial support and sponsorship}

This work is supported from Shanghai Education Committee of Shuguang Plan (18SG32), the National Key Basic Research Program “973 project” (2015CB555400), the General Program from Shanghai Municipal Health Commission (201740249).

\section{Conflicts of interest}

Both authors declared that there are no conflicts of interest.

\section{Ethical approval and consent to participate}

Not applicable.

\section{Consent for publication}

Not applicable.

\section{Copyright}

(C) The Author(s) 2020.

\section{REFERENCES}

1. Venook AP, Papandreou C, Furuse J, de Guevara LL. The incidence and epidemiology of hepatocellular carcinoma: a global and regional perspective. Oncologist 2010;15:5-13.

2. Alazawi W, Cunningham M, Dearden J, Foster GR. Systematic review: outcome of compensated cirrhosis due to chronic hepatitis C infection. Aliment Pharmacol Ther 2010;32:344-55.

3. Berzigotti A, Reig M, Abraldes JG, Bosch J, Bruix J. Portal hypertension and the outcome of surgery for hepatocellular carcinoma in compensated cirrhosis: a systematic review and meta-analysis. Hepatology 2015;61:526-36.

4. Bray F, Ferlay J, Soerjomataram I, Siegel RL, Torre LA, Jemal A. Global cancer statistics 2018: GLOBOCAN estimates of incidence and mortality worldwide for 36 cancers in 185 countries. CA Cancer J Clin 2018;68:394-424.

5. Kaiser K, Mallick R, Butt Z, Mulcahy MF, Benson AB, Cella D. Important and relevant symptoms including pain concerns in hepatocellular carcinoma (HCC): a patient interview study. Support Care Cancer 2014;22:919-26.

6. Chaiteerakij R, Roberts LR. Genetic alterations in advanced HBV-related HCC with portal vein tumor thrombosis: insights from next generation DNA sequencing. J Hepatol 2013;58:1042-4.

7. Zhang XP, Gao YZ, Chen ZH, et al. An eastern hepatobiliary surgery hospital/portal vein tumor thrombus scoring system as an aid to decision making on hepatectomy for hepatocellular carcinoma patients with portal vein tumor thrombus: a multicenter study. Hepatology 2019;69:2076-90

8. Lee HS. Management of patients with hepatocellular carcinoma and extrahepatic metastasis. Dig Dis 2011;29:333-8.

9. Wong R, Frenette C. Updates in the management of hepatocellular carcinoma. Gastroenterol Hepatol (N Y) 2011;7:16-24.

10. Toyoda H, Kumada T, Tada T. Highly sensitive Lens culinaris agglutinin-reactive $\alpha$-fetoprotein: a new tool for the management of hepatocellular carcinoma. Oncology 2011;81:61-5

11. Au JS, Frenette CT. Management of hepatocellular carcinoma: current status and future directions. Gut Liver 2015;9:437-48.

12. Bodzin AS, Busuttil RW. Hepatocellular carcinoma: advances in diagnosis, management, and long term outcome. World J Hepatol 2015;7:1157-67.

13. Liu Y, Wang YR, Dingi GH, et al. Influence of surgical resection of hepatocellular carcinoma(HCC) for hematogenous dissemination of HCC cells and its effect on recurrence and metastasis: 3 years prospective study. Neoplasma 2015;62:635-40.

14. Lacaze L, Scotté M. Surgical treatment of intra hepatic recurrence of hepatocellular carcinoma. World J Hepatol 2015;7:1755-60.

15. Arima K, Chikamoto A, Hashimoto D, et al. Education and imaging. hepatology: hepatocellular carcinoma with duodenal metastasis. $J$ Gastroenterol Hepatol 2015;30:1115.

16. Fang JH, Zhou HC, Zhang C, et al. A novel vascular pattern promotes metastasis of hepatocellular carcinoma in an epithelialmesenchymal transition-independent manner. Hepatology 2015;62:452-65. 
17. Hanley KL, Feng GS. A new VETC in hepatocellular carcinoma metastasis. Hepatology 2015;62:343-5.

18. Portolani N, Coniglio A, Ghidoni S, et al. Early and late recurrence after liver resection for hepatocellular carcinoma: prognostic and therapeutic implications. Ann Surg 2006;243:229-35.

19. Lee SS, Shin HS, Kim HJ, et al. Analysis of prognostic factors and 5-year survival rate in patients with hepatocellular carcinoma: a single-center experience. Korean J Hepatol 2012;18:48-55.

20. Eguchi S, Kanematsu T, Arii S, et al. Recurrence-free survival more than 10 years after liver resection for hepatocellular carcinoma. $\mathrm{Br} J$ Surg 2011;98:552-7.

21. Bruix J. Treatment of hepatocellular carcinoma. Hepatology 1997;25:259-62.

22. Imamura H, Seyama Y, Kokudo N, et al. One thousand fifty-six hepatectomies without mortality in 8 years. Arch Surg 2003;138:1198206.

23. Liu R, Wakabayashi G, Kim HJ, et al. International consensus statement on robotic hepatectomy surgery in 2018. World J Gastroenterol 2019;25:1432-44.

24. Vibert E, Perniceni T, Levard H, Denet C, Shahri NK, Gayet B. Laparoscopic liver resection. Br J Surg 2006;93:67-72.

25. Reich H, McGlynn F, DeCaprio J, Budin R. Laparoscopic excision of benign liver lesions. Obstet Gynecol 1991;78:956-8.

26. Zhou WP, SunZH, Wu MC, et al. The first report of laparoscopic liver lobectomy. J Hepat Surg 1994;2:82. (In Chinese)

27. Chen XP. Expert consensus on laparoscopic hepatectomy (2013 version). J Huazhong Univ Sci Technolog Med Sci 2013;33:791-7.

28. Mise Y, Sakamoto Y, Ishizawa T, et al. A worldwide survey of the current daily practice in liver surgery. Liver Cancer 2013;2:55-66.

29. Lee DH, Kim D, Park YH, Yoon J, Kim JS. Long-term surgical outcomes in patients with hepatocellular carcinoma undergoing laparoscopic vs. open liver resection: A retrospective and propensity score-matched study. Asian J Surg 2020; doi: 10.1016/ j.asjsur.2020.05.028.

30. Takaoka M. A commentary on: "Physical recovery after laparoscopic vs. open liver resection - A prospective cohort study". Int J Surg 2020;74:22.

31. Rao A, Rao G, Ahmed I. Laparoscopic vs. open liver resection for malignant liver disease. A systematic review. Surgeon 2012;10:194201.

32. Memeo R, de'Angelis N, Compagnon P, et al. Laparoscopic vs. open liver resection for hepatocellular carcinoma of cirrhotic liver: a casecontrol study. World J Surg 2014;38:2919-26.

33. El-Gendi A, El-Shafei M, El-Gendi S, Shawky A. Laparoscopic versus open hepatic resection for solitary hepatocellular carcinoma less than $5 \mathrm{~cm}$ in cirrhotic patients: a randomized controlled study. J Laparoendosc Adv Surg Tech A 2018;28:302-10.

34. Takahara T, Wakabayashi G, Beppu T, et al. Long-term and perioperative outcomes of laparoscopic versus open liver resection for hepatocellular carcinoma with propensity score matching: a multi-institutional Japanese study. $J$ Hepatobiliary Pancreat Sci 2015;22:721-7.

35. Yan Y, Cai X, Geller DA. Laparoscopic liver resection: a review of current status. J Laparoendosc Adv Surg Tech A 2017;27:481-6.

36. Milone L, Daskalaki D, Fernandes E, Damoli I, Giulianotti PC. State of the art in robotic hepatobiliary surgery. World J Surg 2013;37:2747-55.

37. Hu L, Yao L, Li X, Jin P, Yang K, Guo T. Effectiveness and safety of robotic-assisted versus laparoscopic hepatectomy for liver neoplasms: a meta-analysis of retrospective studies. Asian J Surg 2018;41:401-16.

38. Giulianotti PC, Coratti A, Angelini M, et al. Robotics in general surgery: personal experience in a large community hospital. Arch Surg 2003;138:777-84.

39. Nota CL, Rinkes IHB, Molenaar IQ, van Santvoort HC, Fong Y, Hagendoorn J. Robot-assisted laparoscopic liver resection: a systematic review and pooled analysis of minor and major hepatectomies. HPB (Oxford) 2016;18:113-20.

40. Idrees K, Bartlett DL. Robotic liver surgery. Surg Clin North Am 2010;90:761-74.

41. Gonzalez-Ciccarelli LF, Quadri P, Daskalaki D, Milone L, Gangemi A, Giulianotti PC. Robotic approach to hepatobiliary surgery. Chirurg 2017;88:19-28.

42. Casciola L, Patriti A, Ceccarelli G, Bartoli A, Ceribelli C, Spaziani A. Robot-assisted parenchymal-sparing liver surgery including lesions located in the posterosuperior segments. Surg Endosc 2011;25:3815-24.

43. Pessaux P, Diana M, Soler L, Piardi T, Mutter D, Marescaux J. Towards cybernetic surgery: robotic and augmented reality-assisted liver segmentectomy. Langenbecks Arch Surg 2015;400:381-5.

44. Zhao ZM, Yin ZZ, Meng Y, Jiang N, Ma ZG, Pan LC, et al. Successful robotic radical resection of hepatic echinococcosis located in posterosuperior liver segments. World J Gastroenterol 2020;26:2831-8.

45. Zhao ZM, Yin ZZ, Pan LC, Hu MG, Tan XL, Liu R. Robotic isolated partial and complete hepatic caudate lobectomy: a single institution experience. Hepatobiliary Pancreat Dis Int 2020; doi: 10.1016/j.hbpd.2020.05.003.

46. Hu M, Chen K, Zhang X, Li C, Song D, Liu R. Robotic, laparoscopic or open hemihepatectomy for giant liver haemangiomas over $10 \mathrm{~cm}$ in diameter. BMC Surg 2020;20:93.

47. Kamarajah SK, Bundred J, Manas D, Jiao LR, Hilal MA, White SA. Robotic versus conventional laparoscopic liver resections: a systematic review and meta-analysis. Scand J Surg 2020; doi: 10.1177/1457496920925637.

48. Ziogas IA, Giannis D, Esagian SM, Economopoulos KP, Tohme S, Geller DA. Laparoscopic versus robotic major hepatectomy: a systematic review and meta-analysis. Surg Endosc 2020; doi: 10.1007/s00464-020-08008-2.

49. Gavriilidis P, Roberts KJ, Aldrighetti L, Sutcliffe RP. A comparison between robotic, laparoscopic and open hepatectomy: a systematic review and network meta-analysis. Eur J Surg Oncol 2020;46:1214-24.

50. Schnitzbauer AA, Lang SA, Goessmann H, Nadalin S, Baumgart J, Farkas SA, et al. Right portal vein ligation combined with in situ 
splitting induces rapid left lateral liver lobe hypertrophy enabling 2-staged extended right hepatic resection in small-for-size settings. Ann Surg 2012;255:405-14.

51. Matayoshi T, Oya Y. Current strategy for management of resistant hypertension. Nihon Rinsho 2011;69:1987-92. 